\title{
Functional Brain Networks: beyond the small-world paradigm
}

\author{
Javier M. Buldú $\hat{u}^{*, * *}$ David Papo ${ }^{* * *}$ Jose A. Pineda ${ }^{* * * *}$ \\ Francisco del Pozo ${ }^{* * * *}$ Stefano Boccaletti ${ }^{* * * *}$ \\ * Complex Systems Group, Universidad Rey Juan Carlos, 28922 \\ Móstoles, Madrid, Spain (e-mail: javier.buldu@urjc.es). \\ ** Laboratory of Biological Networks, Centre for Biomedical \\ Technology, 28923 Pozuelo, Madrid, Spain \\ *** Computational Systems Biology Group, Centre for Biomedical \\ Engineering, 28923, Pozuelo, Madrid, Spain \\ **** Centre for Biomedical Engineering, 28923, Pozuelo, Madrid, \\ Spain
}

\begin{abstract}
This contribution reviews the current state of art comprising the application of Complex Networks Theory to the analysis of functional brain networks. We briefly overview the main advances in this field during the last decade and we explain how graph analysis has increased our knowledge about how the brain behaves when performing a specific task or how it fails when a certain pathology arises. We also show the limitations of this kind of analysis, which have been a source of confusion and misunderstanding when interpreting the results obtained. Finally, we discuss about a possible direction to follow in the next years.
\end{abstract}

Keywords: Brain networks, graph metrics, small-world, functional connectivity.

\section{INTRODUCTION}

Since the two seminal papers of Watts \& Strogatz [Watts and Strogatz (1998)] and Barabási \& Albert [Barabási and Albert (2009)] the understanding of how real networks behave has advanced significantly due, in one hand, to the access to large datasets and, on the other hand, to the analysis of the interplay between structural and dynamical properties of the network of connections. The field of Complex Networks has been extremely active, as indicated by the extensive number of publications and the wide amount of topics covered [Newman (2003); Boccaletti et al. (2006); Costa et al. (2011)]. Biological systems have also been analyzed under this perspective from micro-scales (proteins, genes, neurons, ...), to mesoscales (groups of neurons, genetic pathways, ...) or macroscales (brain networks, ecosystems, ...). In the context of the brain, this kind of approaches have been specially fruitful [Reijneveld et al. (2007); Bullmore and Sporns (2009); Rubinov and Sporns (2010)] due to the fact that, since the brain is the most paradigmatic example of a complex system, its behaviour can only be understood by looking at the whole system simultaneously, in spite of studying their fundamental parts as isolated components. Within this framework, the topology of anatomical and functional brain networks as been analyzed, compared and explained, identifying those nodes with higher relevance

\footnotetext{
* This work was supported by the Spanish Ministry of S\&T [FIS2009-07072] and by the Community of Madrid under project URJC-CM-2010-CET-5006 and the R\&D Program of activities MODELICO-CM [S2009ESP-1691].
}

in the topological structure, the existence of network motifs, or community clusters and evaluating the hierarchy and randomness of this kind of networks. In the case of functional networks, the construction of the network is a problem by itself, since links are placed between regions that are act coordinated and it is extremely difficult to quantify it. In addition, functional correlations between brain sites are always low, what hinders the extraction of the real topology of connections. The existence of weights, causality and time evolution are other factors that make the construction and analysis of functional brain networks a problem that has not been, for the moment, successfully solved. In this contribution we will discuss all this problems in order to have a complete picture of the main drawbacks of this kind of systems and propose the grounds for finding alternative solutions.

\section{COMPLEX NETWORKS THEORY: A VALUABLE TOOL}

\subsection{Understanding functional brain networks}

The analysis of brain networks is the analysis of matrices. Once the brain sites (nodes) and their links (anatomical of functional) have been identified, the information is placed into a matrix as the one shown in Fig. 1. Next, the mathematical analysis of the matrix will give us a wide variety of information, ranging form simple measures, such as the number of connections that each node has, to more complex ones, as the modularity, hierarchy or synchronizability of the network. Finally, in order to have a more qualitative picture, we can project the network into a three-dimensional space, highlighting those nodes 


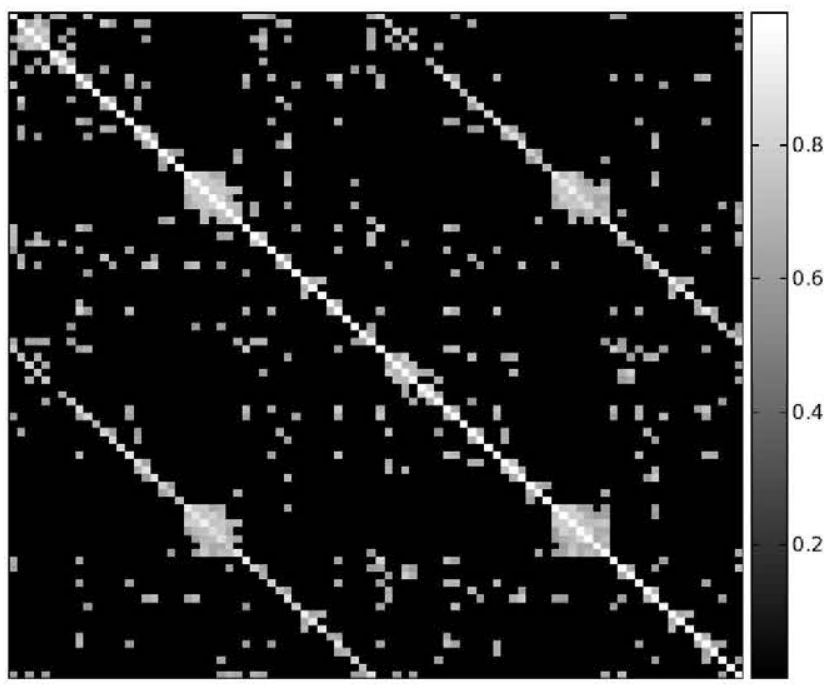

Fig. 1. Example of a resting-state functional connectivity (rsFC) matrix representing correlations of fMRI time series. Resting-state acquisition was performed on a $3 \mathrm{~T}$ General Electric MR scanner (General Electric Healthcare, Farfield, CT), using a whole-body radiofrequency (RF). Scan consisted on 6 minutes of resting state functional MRI with Gradient-Echo EPI sequence $[\mathrm{TR}=3000 \mathrm{~ms} ; \mathrm{TE}=$ Minimum;Flip Angle $=90$ ], yielding to time series of 120 samples. Functional images were preprocessed with SPM8 for slice timing correction and realignment. Next, time courses were band pass filtered [0.01-0.09] Hz. Subject gray matter was parcelled in 88 regions, and time series were averaged within each region. The functional network was obtained performing Pearson linear correlations between all 88 pre-processed time series and keeping the results with pile-03. Components of the depicted matrix represent these correlations after applying absolute threshold of 0.6 .

that play a relevant role in the topology or disregarding those links that do not achieve a certain threshold (see the example of Fig. 2).

Probably, the most known feature about real networks is that the majority of them are "small-world". This property, initially defined in the context of social networks [Milgram (1967)] and lately extended to biological ones [Watts and Strogatz (1998)], is related with the minimum number of steps in order to go from one node to any other of the network. If the average number of steps is much lower than the number of nodes (specifically, if it scales with $\ln (N)$ ) we say that the network has the "small-world property". In addition, if the number of triangles found is much higher than in an equivalent random network (i.e., a network obtained by randomly reshuffling all links of the network) we have a "small-world network".

Concerning the anatomical structure of the brain, complex networks analysis has given us some hints, despite we are still far from a complete knowledge. Studies in different species, such as C. Elegans [Watts and Strogatz (1998)], cats or macaques [Hilgetag et al. (2000)], have revealed common topological properties, such as high clustering and short distance between nodes, i.e., the fingerprint of a small-world (SW) architecture. With regard to the hu- man brain, magnetic resonance imaging [He et al. (2007); Iturria-Medina et al. (2007)] and diffusion spectrum imaging [Hagmann et al. (2008)] have been used in order to obtain the pathways between cortical regions. Up to now, we know that SW property is also present in the human brain together with a truncated power-law degree distribution, the latest indicating the absence of large hubs. Despite the anatomical structure is the substrate of functional brain networks, they do not necessary share common topologies due to the fact that different functional networks arise depending on the task that the brain is performing. Interestingly, it has been shown that the structure of the anatomical network is strongly correlated with the functional brain network in the resting state [Achard et al. (2006)]. The analysis of brain networks obtained from functional MRI (fMRI), electroencephalography (EEG) and magnetoencephalography (MEG) have revealed the functional properties of the brain when performing a given task. Despite there are important differences in the spatial and temporal resolution of these methods, all of them have shown the SW property [Watts and Strogatz (1998)] of the brain networks [Bullmore and Sporns (2009)] is related to short topological distance between any pair of nodes (due to the existence of long-range connections) and high clustering coefficient (due to dense local connections). Small-worldness of brain networks seems to play a crucial role in complex dynamical processes such as information transmission, pattern recognition or learning [Simard et al. (2005)]. Other studies have gone one step beyond the SW configuration and have quantified the importance of hubs [Sporns et al. (2007)], unveiled the existence of characteristic network motifs [Sporns and Kötter (2004)], and also detected the appearance of community structures [Hagmann et al. (2008)], which are related to the segregated organization of the brain.

\subsection{From a healthy to an impaired brain}

Apart from the understanding of the topological properties of this kind of networks, one of the promising challenges of the application of graph theoretical analysis is the identification of network signatures of impairments in order to understand the evolution and consequences of different brain diseases, and to identify individuals susceptible (or with a high risk) of developing a certain pathology. In this sense, there have been revealing studies in the characterization of Alzheimer disease (AD), schizophrenia or epilepsy. MEG and EEG on patients with Alzheimer have shown that the disease is related to a loss of the SW properties of the functional network [Stam et al. (2007); Supekar et al. (2008); Stam et al. (2009)], specifically with an increase of the mean path length between nodes which leads to a partition of the network if a certain correlation threshold is considered [Stam et al. (2007)]. Interestingly, the resting state functional network of $\mathrm{AD}$ patients shows a decrease of the clustering coefficient that is associated to an evolution towards a random topology as a consequence of the deterioration of the local synchrony [Supekar et al. (2008)]. The increase of randomness, together with a loss of the network modularity, is also reported in mild cognitive impairment [Buldu et al. (2011)], a disease with a high rate of conversion into $\mathrm{AD}$. In the case of schizophrenia, similar studies have detected an abnormal configuration 
of the anatomical network, consisting on a reduction of the hierarchical structure of the network, an enhance of the mean shortest path and a loss of frontal hubs [Basset et al. (2008)]. Again, the onset of the disease is related to an evolution towards random topologies. Nevertheless, it is worth noting that not all brain diseases are associated with the same topological deterioration. In epilepsy, for example, the enhancement of the synchronized activity during seizures leads to an increase of the clustering coefficient of the network, which reduces the randomness of the functional network and increases the SW network property [Ponten et al. (2007)]. In this case, the network is more regular in patients with epilepsy than in healthy individuals, which indicates that functional brain networks are a trade-off between regular and random structures.

\section{LIMITATIONS OF GRAPH ANALYSIS}

Neverheless, we have to keep in mind that $\mathrm{f}$ unctional brain network are not real. This is the main drawback of this kind of networks, since the connections between nodes (brain sites) are not physical. Therefore, the construction and analysis of functional brain network relies in three crucial steps, all of them with weak points: 1) the measurement of the brain activity, 2) the extraction of the correlation between brain sites, 3 ) the analysis of the networks obtained. In the following, we will discuss which are the main limitations of each one of these steps.

\subsection{Experimental limitations: how to analyze what we can not observe}

The activity of the human brain is a consequence of the coordinated dynamics of around $10^{11}$ neurons, some of them with more that $10^{3}$ synaptic connections. Nevertheless, the resolution of the available technology in order to measure brain activity, namely, magnetoencephalography (MEG), electroencephalography (EEG) and functional magnetic resonance imaging (fMRI) is several orders of magnitude lower both in time and spatial scales. These limitations are related with the following key drawbacks:

(1) Despite the high spatial resolution of fMRI (when compared with EEG or MEG), we are only able to measure the activity of large groups of neurons which, in turn, could operate with complex patterns or even out of synchrony. This spatial averaging filters dramatically the information about what are the real dynamical and connectivity patterns of the brain.

(2) The higher temporal resolution of EEG and MEG techniques may not be able to fully res olve the dynamical spectrum of brain networks. Nowadays there is an intense discussion about how high frequencies (in the order of hundreds of $H z$ ) are related with brain activity.

(3) There is not a clear division of the brain sites or, at least, the measurement of each sensor (in EEG and MEG) is not constraint to a specific brain site. In this way, one sensor could be measuring the activity of two, or more, group of neurons having completely different dynamics.

(4) There is a high variability in the results. It is impossible to reproduce the same experiment, under the same conditions and with the same results.

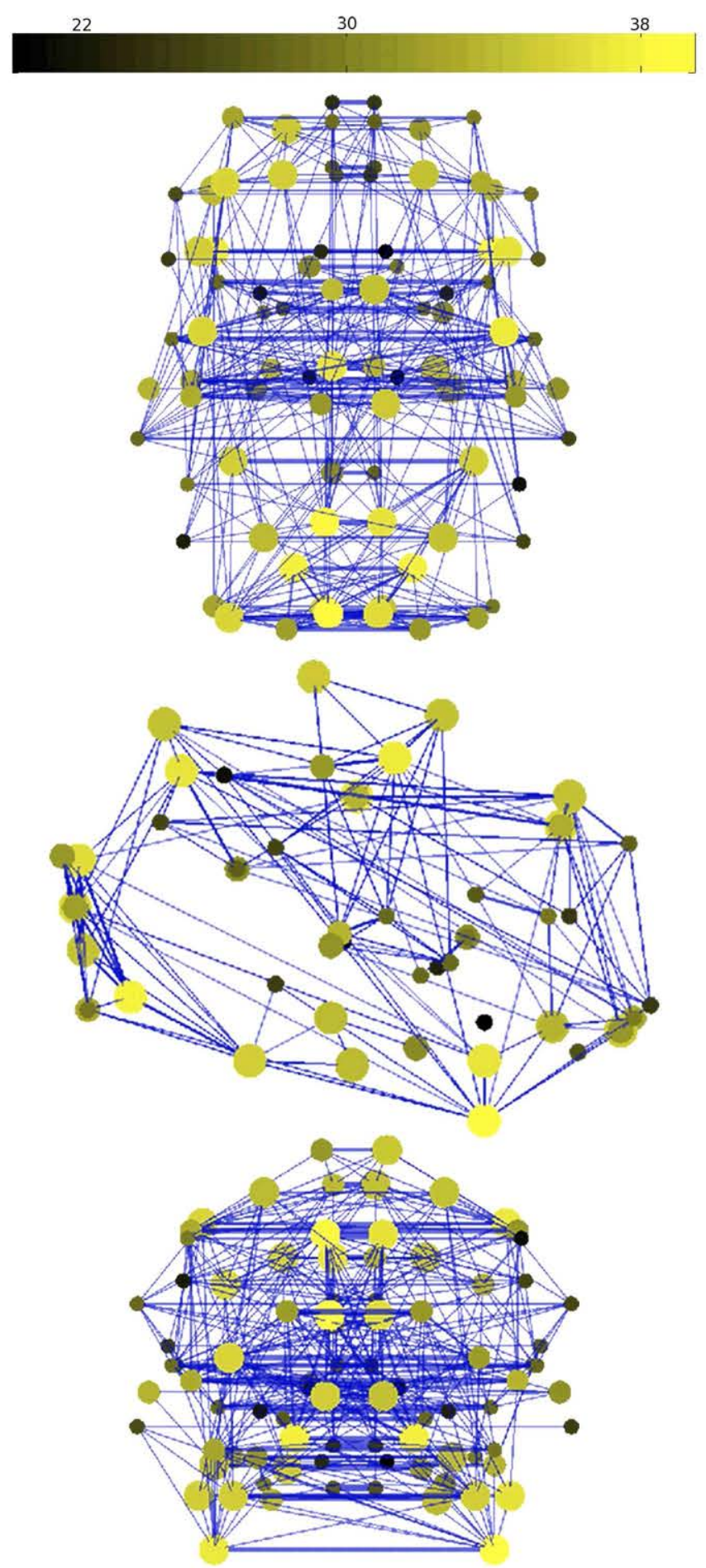

Fig. 2. Axial, sagittal and coronal views of the rsFC network depicted in Fig. 1, where links (blue) are depicted in three different widths attending to the link weights. Circles represent the nodes of the rsFC network, which are sized attending to the node strength within the network.

\subsection{Network limitations: how to obtain functional brain} networks

Let us suppose that we are able to overcome the limitations explained in the previous subsection. We can imagine that we have accurately measured the activity (magnetic or electrical) of a certain number of brain sites. The next step 
is to extract the topology of connections between nodes, i.e., to measure the correlated activity between pairs of brain sites. Again, we are facing a number of problems that hinder the extraction of the network topology:

(1) The links of the network are weighted. There exist a wide spectrum of values for the correlation between brain sites and the classical reduction to a binary network of connections ( 1 if correlation is above certain threshold and 0 otherwise) filters a lot of information contained in the correlation values.

(2) Links have a certain directionality. Another classical reduction is to consider an undirected (symmetric) matrix of connections, since the analysis of the network is much more simple. Nevertheless, functional networks are directed by definition, since physical connections between brain sites have directionality. Therefore, we are disregarding, again, valuable information about the structure of connections.

(3) Correlations between nodes change in time. The most extended methodology is to obtain correlations (i.e., link weights) is to analyze a pair of time series, obtaining a unique value for the whole series. This procedure is necessary in the case of fMRI, due to the low temporal resolution, but should be avoided in the case of EEG or MEG.

(4) We have a diverse group of measures of the correlated activity [Pereda et al. (2007)], in some cases leading to similar results and, in others, completely different. Generalized synchronization, phase lag index, phase locking value, synchronization likelihood, wavelet coherence, each of them measuring a particular property of the time series, which hampers the comparison of results obtained with different measures.

\subsection{The risk of misinterpreting}

The complex network analysis is the last, but not the least, step of these kind of studies. In principle, the experimental restrictions should be the limit to the network analysis and, due to the high number of drawbacks exposed above, the conclusions of these kind of studies should avoid strong statements. Nevertheless, it is not the case of many studies and, in some cases, conclusions are misleading, since we have to be extremely careful with the interpretation of, at least, the following points:

(1) The term small-world has been widely used without knowledge of its implications. In many cases a functional network is said to be small-world only by accomplishing that the average path length $d$ is much lower than the number of nodes of the network $N$, i.e., $d<<N$. If it is the case, network are said to be optimized from the point of view of the number of steps that a given message has to make to reach any other node of the network. To be rigorous, the shortest path length should decrease with $N$ as $\log (N)$, nevertheless, in many cases functional networks are so small that it is difficult to appreciate this scaling law. The case of a regular two-dimensional network is a simple counter example. If we take a regular lattice of $100 \times 100$ (i.e., $N=10000$ ), the average shortest path goes with $d \propto N^{1 / 2}$, i.e., we have a difference between $d$ and $N$ of two orders of magnitude. The same reasoning applies to other topological measures such as clustering or modularity as recently explained in Ref. [Henderson and Robinson (2011)].

(2) Another term that has caused confusion is the network efficiency. Introduced by Latora et al. [Latora and Marchiori (2001)], the global efficiency of a network is related with the inverse of the shortest path between all nodes of the network. It is an interesting value in order to evaluate the average distance between any two nodes, but it is not an indicator of how efficient the functional network is, at least in the terms of energy vs. task performance. In addition, it is fearless to affirm that efficiency could reveal an optimal distribution of nodes with regard to the transmission of information, since it does not consider other physiological processes that are crucial to an optimal transfer of information, such as delays, redundancy or self-loops.

(3) Synchronizability has not been tested as a suitable term to measure the ability of the network to synchronize. In Complex Networks Theory, synchronizability is measured by computing the second eigenvalue of the Laplacian Matrix [Boccaletti et al. (2006)]. Nevertheless, this parameter only applies to networks with identical nodes, which is not the case of functional networks. Despite this limitation, synchronizability is a parameter widely used in functional networks analysis.

(4) We have to be extremely careful when comparing networks. Functional networks obtained with different techniques will lead to different results. Even networks of the same individual measured at different time intervals may diverge [Deuker et al. (2009)]. There still exists a need for ground studies on the reproducibility of functional networks and the combination of information obtained from different experimental techniques.

\section{NEXT GOALS, NEXT ANALYSIS}

Therefore, due to the particular properties of functional brain networks, we have to avoid classical analysis of complex networks, and concentrate in the development of new and specific network parameters in order to characterize the structure of this kind of networks. Specifically, we should focus in three main points:

(1) The definition of correct measures in order to evaluate the correlated activity between brain sites. Depending on the task performed, this measures should include directionality, weights and delay. In addition, they should undergo some kind of normalization to allow comparison with networks of different individuals.

(2) We have to define parameters that are able to capture the dynamical nature of functional networks, since we are not dealing with static networks. This problem is of special interest in cognitive tasks, were correlations between nodes are modified at scales of milliseconds. We should not only compute the classical network parameters and follow their evolution in time, but to define new parameters that capture the ability of the network to change or adapt to external perturbations.

(3) We have to quantify the reproducibility of graph metrics. Up to now, there are few works that measure 
changes in the network topology when the experiment is repeated under the same conditions. Are complex networks consistent [Uchida et al. (2004)]? (i.e., do they behave in the same way when repeating the same task?) Until we do not answer to this question, we will not be able to stress the results obtained with complex networks analysis.

\section{CONCLUSION}

In the current contribution we have made a brief overview of the main advantages and disadvantages of the use of Complex Networks Theory for the analysis of functional brain networks. As we have seen, despite the technical limitations, we have already obtained fruitful results concerning the operation and dysfunction of brain networks. Nevertheless, we still must to make an effort to define the most adequate network parameters in order to better fit to the special features kind of networks and overcome the unavoidable (technical) limitations of this kind of approximation to the analysis of the brain dynamics.

\section{ACKNOWLEDGEMENTS}

We thank Fernando Maestú (C.T.B.-U.P.M.) and Antoni Pons (U.P.C.) for fruitful discussions.

\section{REFERENCES}

Achard, S., Salvador, R., Whitcher, B., Suckling, J., and Bullmore, E. (2006). A resilient low-frequency, smallworld human brain functional network with highly connected association cortical hubs. J. Neurosci, 26, 63-72.

Barabási, A.L. and Albert, R. (2009). Emergence of scaling in random networks. Science, 286, 509.

Basset, D., Bullmore, E., Verchinski, B., Mattay, V., Weinberger, D., , and A., M.L. (2008). Hierarchical organization of human cortical networks in health and schizophrenia. J. of Neuroscience, 28, 9239-9248.

Boccaletti, S., Latora, V., Moreno, Y., Chavez, M., and Hwang, D. (2006). Complex networks: Structure and dynamics. Physics Reports, 424(4-5), 175-308.

Buldu, J., Bajo, R., Maestu, F., Castellanos, N., Leyva, I., Gil, P., Sendiña Nadal, I., Almendral, J.A., Nevado, A., del Pozo, F., and Boccaletti, S. (2011). Reorganization of functional networks in mild cognitive impairment. PLOS ONE, 6, e19584.

Bullmore, K. and Sporns, O. (2009). Complex brain networks: graph theoretical analysis of structural and functional systems. Nature, 10, 186.

Costa, L.F., Oliveira, O.N., Travieso, G., Rodrigues, F.A., Villas Boas, P.R., Antiqueira, L., Viana, M.P., and da Rocha, L.E.C. (2011). Analyzing and modeling realworld phenomena with complex networks: A survey of applications. Science, 60, 329-412.

Deuker, L., Bullmore, E.T., Smith, M., Christensen, S., Nathan, P.J., Rockstroh, B., and Bassett, D.S. (2009). Reproducibility of graph metrics of human brain functional networks. Neuroimage, 47, 1460-1468.

Hagmann, P., Kurant, M., Gigandet, X., Thiran, P., Wedeen, V., Meuli, R., and Thiran, J. (2008). Mapping human whole-brain structural networks with diffusion mri. PLOS Biology, 6, e159.
He, Y., Chen, Z.J., and Evans, A.C. (2007). Small-world anatomical netowrks in the human brain revealed by cortical thickness from mri. Cereb Cortex, 17, 24072419.

Henderson, J. and Robinson, P. (2011). Geometric effects on complex network structure in the cortex. Phys. Rev. Lett., 107, 018102.

Hilgetag, C., Burns, G., O'Neill, M., Scannell, J., and Young, M. (2000). Anatomical connectivity defines the organization of clusters of cortical areas in the macaque monkey and the cat. Phil. Trans. R. Soc. Lond. B, 355, 91-110.

Iturria-Medina, Y., Canales-Rodriguez, E., Melie-Garcia, L., Valdes-Hernandez, P., Martinez-Montes, E., AlemanGmez, Y., and J.M., S.B. (2007). Characterizing brain anatomical connections using diffusion weighted mri and graph theory. Neuroimage, 36, 645-660.

Latora, V. and Marchiori, M. (2001). Efficient behavior of small-world networks. Phys. Rev. Lett., 87, 198701.

Milgram, S. (1967). The small world problem. Psychol. Today, 1, 61-67.

Newman, M.E.J. (2003). The Structure and Function of Complex Networks. SIAM Review, 45(2), 167-256.

Pereda, E., Quiroga, R., and Bhattacharya, J. (2007). Nonlinear multivariate analysis of neurophysiological signals. Progress in neurobiology, 77, 1-37.

Ponten, S., Bartolomei, F., and Stam, C. (2007). Smallworld networks and epilepsy: graph theoretical analysis of intracerebrally recorded mesial temporal lobe seizures. Clinical Neurophysiology, 118, 918-927.

Reijneveld, J.C., Ponten, S.C., Berendse, H.W., and Stam, C.J. (2007). The application of graph theoretical analysis to complex networks in the brain. Clinical neurophysiology, 118, 2317-31.

Rubinov, M. and Sporns, O. (2010). Complex network measures of brain connectivity: uses and interpretations. Neuroimage, 52, 1059-1069.

Simard, D., Nadeau, L., , and Kr'oger, H. (2005). Fastest learning in small-world neural networks. Phys. Lett. A, $336,8-15$.

Sporns, O. and Kötter, R. (2004). Motifs in brain networks. PLoS Biol., 2, 1910-1918.

Sporns, O., Tononi, G., and Kötter, R. (2007). Identification and classification of hubs in brain networks. PLoS ONE, 2, e1049.

Stam, C.J., de Haan, W., Daffertshofer, A., Jones, B.F., Manshanden, I., van Cappellen, Montez, T., Verbunt, J.P.A., de Munck, J.C., van Dijk, B.W., Berendse, H.W., and Scheltens, P. (2009). Graph theoretical analysis of magnetoencephalographic functional connectivity in Alzheimer's disease. Brain, 132(1), 213-224.

Stam, C.J., Jones, B.F., Nolte, G., Breakspear, M., and Scheltens, P. (2007). Small-world networks and functional connectivity in Alzheimer's disease. Cereb Cortex, $17,92-99$.

Supekar, K., Menon, V., Rubin, D., Musen, M., and Greicius, M.D. (2008). Network analysis of intrinsic functional brain connectivity in alzheimers disease. PLoS Comput. Biol., 4, e1000100.

Uchida, A., McAllister, R., and Roy, R. (2004). Consistency of nonlinear system response to complex drive signals. Phys. Rev. Lett, 93, 244102. 
Watts, D.J. and Strogatz, S.H. (1998). Collective dynamics of 'small-world' networks. Nature, 393(6684), 440-442. 